\title{
Delayed aortoesophageal and tracheoesophageal fistulas secondary to foreign body ingestion: a case report
}

\author{
Zaili Zhang ${ }^{1 \#}$, Zhenglun Yu ${ }^{2 \#}$, Wenfei Tan $^{1}$, Kaixi Liu ${ }^{3}$ \\ ${ }^{1}$ Department of Anesthesiology, the First Hospital of China Medical University, Shenyang, China; ${ }^{2}$ Department of Thoracic Surgery, the First \\ Hospital of China Medical University, Shenyang, China; ${ }^{3}$ Department of Anesthesiology, Peking University Third Hospital, Beijing, China \\ \#These authors contributed equally to this work. \\ Correspondence to: Wenfei Tan. Department of Anesthesiology, the First Hospital of China Medical University, Nanjingbei Street 155, Shenyang \\ 110001, China. Email: winfieldtan@hotmail.com.
}

\begin{abstract}
Ingestion of a foreign body $(\mathrm{FB})$ is a common condition with a few potentially life-threatening complications, including esophageal perforation (EP), aortoesophageal fistula (AEF), mediastinal infection, and tracheoesophageal fistula (TEF). In this case, a patient who accidentally ingested a duck bone gradually experienced all of the above complications. To resolve the symptom of difficulty swallowing, the patient underwent emergency treatment for removal of the esophageal FB via endoscopic surgery. Under endoscopy, esophageal mucosal injuries were present, but no other abnormalities, such as active bleeding, were observed. However, the patient returned to our hospital a week later with symptoms of vomiting and black stool and received the diagnosis of EP, AEF and mediastinal infection. Two days later, he vomited 1,000-2,000 mL of blood after experiencing sudden severe chest pain. Then, thoracic endovascular aortic repair (TEVAR) and mediastinal drainage with video-assisted thoracoscopic surgery (VATS) were performed under emergency general anesthesia. Additionally, the patient underwent esophageal stent implantation when TEF was confirmed by tracheal computed tomography (CT). The patient was treated with anti-infective therapy throughout the treatment process. Finally, he recovered and was able to tolerate a liquid diet. Comprehensive evaluation and multidisciplinary cooperation are all very important for the treatment of esophageal foreign bodies and complications.
\end{abstract}

Keywords: Aortoesophageal fistula (AEF); tracheoesophageal fistula (TEF); case report

Submitted Mar 09, 2021. Accepted for publication May 02, 2021.

doi: 10.21037/apm-21-562

View this article at: http://dx.doi.org/10.21037/apm-21-562

\section{Introduction}

Ingestion of a foreign body (FB) is a common condition but can has the potential for life-threatening complications, including esophageal perforation (EP), aortoesophageal fistula (AEF), mediastinal infection, and tracheoesophageal fistula (TEF). Each complication may have catastrophic results, and the treatment plans often present a degree of difficulty and even require multidisciplinary cooperation. In this case, we describe the course of a patient who experienced all of the above complications over a few weeks, which is rare and may provide clinicians with some experience and lessons for treating these complications. We present the following case in accordance with the CARE reporting checklist (available at https://apm.amegroups. com/article/view/10.21037/apm-21-562/rc).

\section{Case presentation}

On May 7, 2020, a 46-year-old male patient came to the First Hospital of China Medical University for treatment due to swallowing a duck bone by mistake $24 \mathrm{~h}$ prior. To resolve the symptom of difficulty swallowing, he underwent emergency treatment for removal of the esophageal FB via endoscopic surgery. The elongated FB (approximately $3 \mathrm{~cm}$ ) 

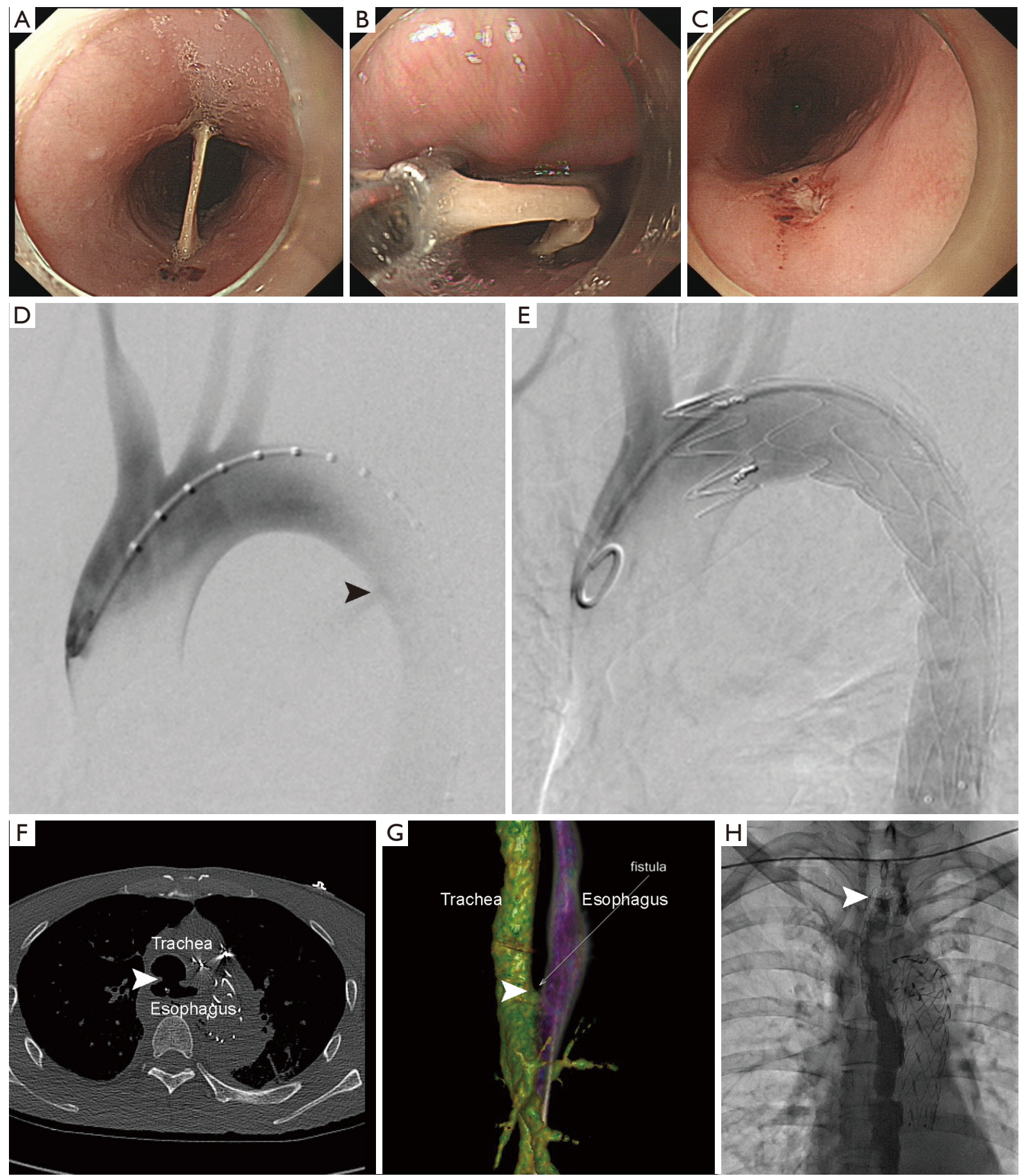

Figure 1 Typical images of the treatment process. (A) An FB is observed to be supported at both ends of the esophageal cross-section. (B) Near image of the FB. (C) The FB was completely removed, and mucosal injury was observed. (D) Aortic rupture viewed by angiography (black arrow). (E) The aortic stent was successfully implanted. (F) and (G) show that TEF was confirmed by tracheal CT (white arrow). (H) An esophageal stent was successfully placed (white arrow). FB, foreign body; TEF, tracheoesophageal fistula; CT, computed tomography. 

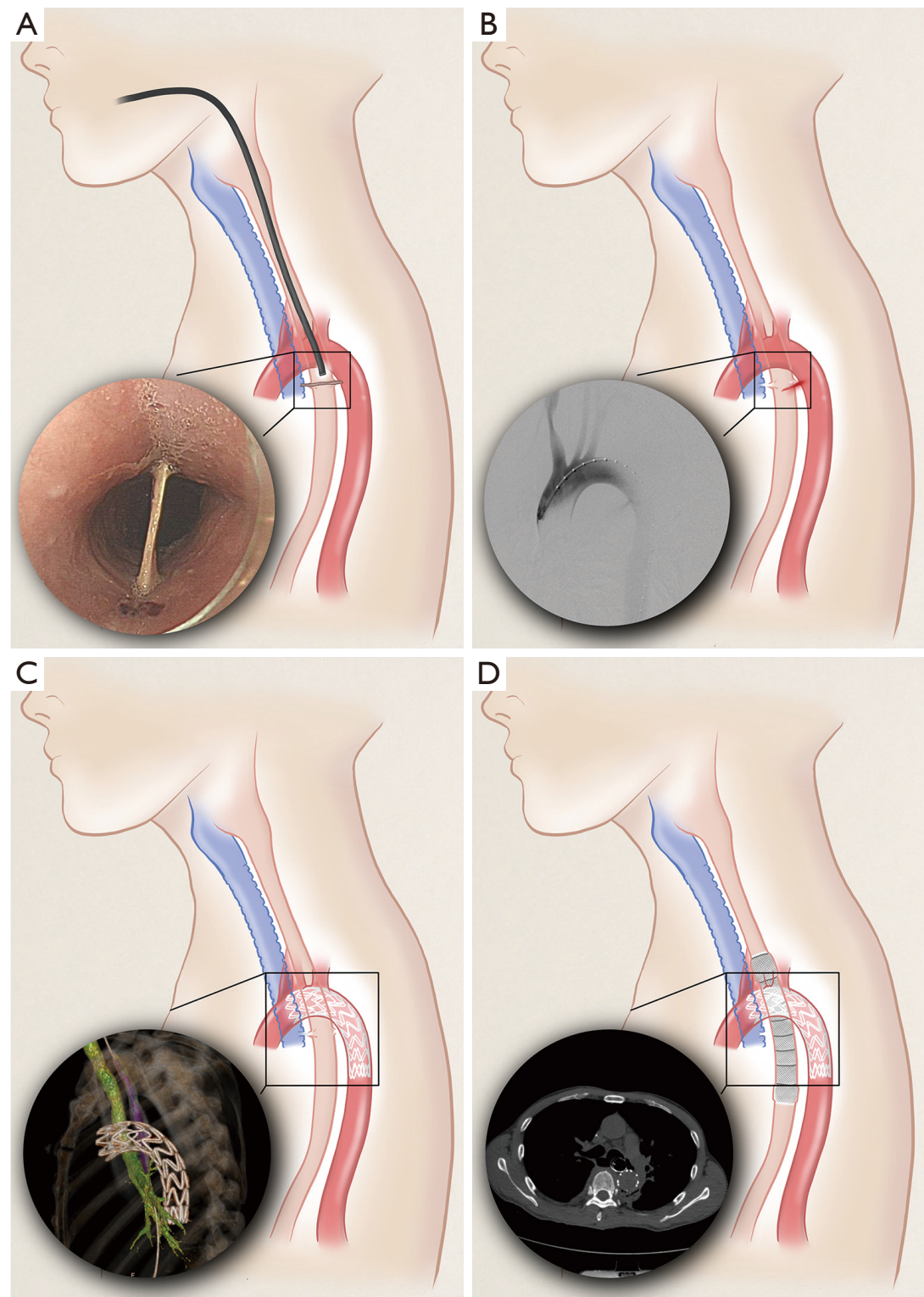

Figure 2 Flowchart of the entire treatment process. (A) The FB was supported at both ends of the esophageal cross-section. (B) Aortic rupture was observed. (C) An aortic stent was successfully implanted. (D) An esophageal stent was successfully placed. FB, foreign body.

(Figure 1) was located in the esophagus $30 \mathrm{~cm}$ from the incisors and was observed to be supported at both ends of the esophageal cross-section (Figure 1A, Figure 2A). The FB was gently loosened with a grasper under gastroscopic guidance and then removed completely without secondary damage (Figure 1B). Under endoscopy, esophageal mucosal injuries were present, but no other abnormalities, such as active bleeding, were observed (Figure 1C). The patient was required to avoid food and drink for $2 \mathrm{~h}$, ingest liquid food for 3 days and then eat soft foods for 1 week; he was also told to see the physician promptly if he had symptoms such as abdominal pain, hematemesis, and black stool. The patient began to ingest liquid food on the second day, but he developed black stools after one week of this liquid diet.

On May 19, 2020, the patient returned to our hospital with symptoms of vomiting and black stool and received a diagnosis of EP, AEF, and mediastinal infection. The patient received intravenous fluid rehydration and anti-infection (imipenem-cilastatin sodium) treatment. On May 21, 2020, he vomited 1,000-2,000 $\mathrm{mL}$ of blood after experiencing 
sudden severe chest pain and subsequently underwent thoracic endovascular aortic repair (TEVAR) and videoassisted thoracoscopic surgery (VATS) under emergency general anesthesia. Angiography revealed a rupture that was visible at the small arch approximately $4 \mathrm{~cm}$ distal to the origin of the left subclavian artery, and a large amount of contrast medium was ejected (Figure 1D, Figure 2B). Finally, the vascular surgeon succeeded in placing the covered stent (Valiant Captiva-26-26-150, Medtronic, USA) from the aortic arch at the left subclavian artery level to the distal end of the descending aorta (Figure 1E, Figure 2C). Approximately $200 \mathrm{~mL}$ of a yellow-green effusion was observed on the right side of the chest under thoracoscopy and subsequently removed by aspiration. Opening the mediastinal pleura, the thoracic surgeon observed a large amount of yellow-white purulent fluid and dark bloody fluid exudation and finally repeated flushing and placement of the drainage tube.

From May 22 to 26, 2020, the patient's condition gradually stabilized after anti-infection (imipenem-cilastatin sodium), intravenous nutrition and other treatments in the intensive care unit (ICU). When the jejunal nutrition tube was placed in the patient on May 27, 2020, the guide wire misentered the trachea several times, and TEF was suspected. TEF was later confirmed by tracheal CT $(3 \mathrm{~cm}$ $\times 2 \mathrm{~cm}$ in the main bronchus) (Figure $1 F, 1 G$ ). As the scope of TEF continued to expand and further treatment was continued, the patient underwent esophageal stent (MTN20-140 mm, Micro-Tech, Nanjing) implantation on June 24, 2020, and the operation was successful (Figure 1H, Figure 2D). The patient continued to receive anti-infective therapy (piperacillin-tazobactam sodium). On July 3, 2020, the patient was discharged from the hospital in stable condition and was able to tolerate liquid food.

The patient reported horrible experiences caused by the complications of the penetrating FB and expressed gratitude to the doctors for the treatment process.

All procedures performed in studies involving human participants were in accordance with the ethical standards of the institutional and/or national research committee(s) and with the Declaration of Helsinki (as revised in 2013). Written informed consent was obtained from the patient for publication of this case report and accompanying images. A copy of the written consent is available for review by the editorial office of this journal.

\section{Discussion}

In rare situations, EP can be caused by an FB. Assessing the extent of esophageal injuries after removal of the FB is an important issue for doctors. In one study, multidetector computed tomography (MDCT) was used to grade esophageal injuries, which indicated a potential risk of AEF when the distance between the FB and the aorta was less than or equal to $2 \mathrm{~mm}$ (1). AEF is a serious, life-threatening complication with a very low survival rate and requires multidisciplinary management; currently, there are no widely accepted guidelines for treatment (2). If the patient has a history of an esophageal FB and the typical symptoms of the Chiari triad (3), such as dysphagia, chest pain, a sentinel episode of hematemesis, and fatal hemorrhage, early diagnosis by MDCT and active surgical treatment are expected to improve the survival rate of AEF. TEF is another serious complication caused by foreign bodies in the esophagus and yields the typical symptom of cough after swallowing. In addition to symptomatic treatment, such as anti-inflammatory and esophageal shunts, active surgical treatment should also be performed after the patient has been weaned off the ventilator and has a good cough reflex (4). Compared with open surgery, VATS has become more popular in recent years (5). Interventional stenting is another approved treatment method (6) that has achieved certain efficacy in the treatment of TEF (7).

In this case, the AEF and TEF were delayed after the FB was fully removed. It is possible that the local infection caused by the FB further aggravated the injury to the esophagus, leading to delayed complications. Computed tomography (CT) and endoscopy did not reveal EP. Could re-examination by CT after endoscopic surgery, prolonging the period of avoiding food and other strategies have avoided such delayed complications? Unfortunately, we did not re-perform CT after the removal of the FB. Additionally, we seriously considered stent placement, but the patient was in such a poor state of infection and nutrition that radical treatment was not suitable. We expect that further radical surgery will be performed when the patient's infection and nutrition condition have improved.

In conclusion, this case may provide clinicians with some experience and lessons. The patient's history, symptoms, experience with endoscopy, early diagnosis of MDCT, grading of esophageal injuries, active surgical treatment and multidisciplinary cooperation are all very important for the treatment of esophageal foreign bodies and complications.

\section{Acknowledgments}

Funding: None. 


\section{Footnote}

Reporting Checklist: The authors have completed the CARE reporting checklist. Available at https://apm.amegroups. com/article/view/10.21037/apm-21-562/rc

Peer Review File: Available at https://apm.amegroups.com/ article/view/10.21037/apm-21-562/prf

Conflicts of Interest: All authors have completed the ICMJE uniform disclosure form (available at https://apm. amegroups.com/article/view/10.21037/apm-21-562/coif). The authors have no conflicts of interest to declare.

Ethical Statement: The authors are accountable for all aspects of the work and ensuring that questions related to accuracy or integrity are appropriately investigated and resolved. Consent for publication and any accompanying images were obtained from the patient. All procedures performed in studies involving human participants were in accordance with the ethical standards of the institutional and/or national research committee(s) and with the Declaration of Helsinki (as revised in 2013). Written informed consent was obtained from the patient. Written informed consent was obtained from the patient for publication of this case report and accompanying images. A copy of the written consent is available for review by the editorial office of this journal.

Open Access Statement: This is an Open Access article distributed in accordance with the Creative Commons Attribution-NonCommercial-NoDerivs 4.0 International License (CC BY-NC-ND 4.0), which permits the non- commercial replication and distribution of the article with the strict proviso that no changes or edits are made and the original work is properly cited (including links to both the formal publication through the relevant DOI and the license). See: https://creativecommons.org/licenses/by-nc-nd/4.0/.

\section{References}

1. Wei Y, Chen L, Wang Y, et al. Proposed management protocol for ingested esophageal foreign body and aortoesophageal fistula: a single-center experience. Int J Clin Exp Med 2015;8:607-15.

2. Zeng L, Shu W, Ma H, et al. Aortic injury caused by esophageal foreign body-case reports of 3 patients and literature review. Medicine (Baltimore) 2020;99:e20849.

3. Rispoli P, Bertoldo U, Oliaro A, et al. Two-stage treatment of a secondary aortoesophageal fistula after thoracic endovascular aneurysm repair. J Cardiovasc Surg (Torino) 2012;53:531-5.

4. Santosham R. Management of Acquired Benign Tracheoesophageal Fistulae. Thorac Surg Clin 2018;28:385-92.

5. Daniel SJ, Smith MM. Tracheoesophageal fistula: open versus endoscopic repair. Curr Opin Otolaryngol Head Neck Surg 2016;24:510-15.

6. Hürtgen M, Herber SCA. Treatment of malignant tracheoesophageal fistula. Thorac Surg Clin 2014;24:117-27.

7. Hovde $\varnothing$, Haugen Lie $\varnothing$, Johansson PA, et al. Iatrogenic esophago-tracheal fistula: challenges in diagnosis and management. World J Gastroenterol 2013;19:594-6.
Cite this article as: Zhang Z, Yu Z, Tan W, Liu K. Delayed aortoesophageal and tracheoesophageal fistulas secondary to foreign body ingestion: a case report. Ann Palliat Med 2022;11(2):827-831. doi: 10.21037/apm-21-562 